\title{
The breathing-mode giant monopole resonance and the surface compressibility in the relativistic mean-field theory
}

\author{
M.M. Sharma \\ Physics Department, Kuwait University, Kuwait 13060
}

\begin{abstract}
The breathing-mode isoscalar giant monopole resonance (GMR) is investigated using the generator coordinate method within the relativistic mean-field (RMF) theory. Employing the Lagrangian models of the nonlinear- $\sigma$ model $(\mathrm{NL} \sigma)$, the scalarvector interaction model (SVI) and the $\sigma-\omega$ coupling model (SIGO), we show that each Lagrangian model exhibits a distinctly different GMR response. Consequently, Lagrangian models yield a different value of the GMR energy for a given value of the nuclear matter incompressibility $K_{\infty}$. It is shown that this effect arises largely from a different value of the surface incompressibility $K_{\text {surf }}$ inherent to each Lagrangian model, thus giving rise to the ratio $K_{\text {surf }} / K_{\infty}$ which depends upon the Lagrangian model used. This is attributed to a difference in the density dependence of the meson masses and hence to the density dependence of the nuclear interaction amongst various Lagrangian models. The sensitivity of the GMR energy to the Lagrangian model used and thus emergence of a multitude of GMR energies for a given value of $K_{\infty}$ renders the method of extracting $K_{\infty}$ on the basis of interpolation amongst forces as inappropriate. As a remedy, the need to 'calibrate' the density dependence of the nuclear interaction in the RMF theory is proposed.
\end{abstract}

Key words: Relativistic mean-field theory, nonlinear- $\sigma$ model, scalar-vector interaction model SVI, $\sigma-\omega$ coupling model SIGO, density dependence of meson masses, generator coordinate method, breathing-mode giant monopole resonance, incompressibility of nuclear matter, surface incompressibility.

PACS: 21.30.Fe, 21.60.Jz, 21.65.-f, 24.30.Cz

Email address: sharma@kuc01.kuniv.edu.kw (M.M. Sharma). 


\section{Introduction}

The compressibility of nuclear matter has been a matter of exploration and discussion for a long time. The incompressibility or the compression modulus is a fundamental property of the nuclear matter. It constitutes a cardinal point on the equation of state (EOS) of the nuclear matter. Defined as the second derivative of the EOS of the nuclear matter at the saturation density, it is also important for astrophysical phenomena such as supernova explosion and structure of neutron stars [1,2,3].

The breathing-mode isoscalar giant monopole resonance (GMR) is a principal source of information on the (in)compressibility of nuclei and nuclear matter [4,5,6]. Nuclei undergo radial density oscillations about the equilibrium point in this mode. It is a small-amplitude collective motion wherein the nucleus participates as a whole. The isoscalar GMR is well-established experimentally and has been studied in a large number of nuclei from heavy to light mass regions in various laboratories around the world $[7,8,9,10]$. Recent measurements [11,12 have brought in improved precision in providing better information on the breathing-mode GMR.

Experimental determination of the incompressibility of nuclear matter has entailed using a liquid-drop model type expansion of the incompressibility $K_{A}$ of a nucleus in terms of a bulk and finite-size components [7]10. Experimental data on the breathing-mode isoscalar GMR on a large number of nuclei was used [7]. Attempts were made to extract the nuclear matter incompressibility $K_{\infty}$ using the data on $\mathrm{Sn}$ and $\mathrm{Sm}$ isotopes obtained with considerable precision [10]. However, the correlation between the Coulomb term and $K_{\infty}$ hinders extraction of the incompressibility of the nuclear matter [13].

With the difficulty of extracting $K_{\infty}$ directly using the experimental data, theoretical approach based upon interpolation amongst Skyrme and Gogny type interactions as proposed by Blaizot [4]14] has been extensively employed. It has required reproducing experimental breathing-mode GMR energies of nuclei using an interaction with an appropriate value of $K_{\infty}$ in self-consistent Hartree-Fock and random-phase approximation (RPA) calculations [14]. In this scheme, the GMR energy of ${ }^{208} \mathrm{~Pb}$ has been shown to be reproduced with forces with $K_{\infty} \sim 220 \mathrm{MeV}$. On the other hand, the RPA calculations with force(s) which reproduce the GMR energy of ${ }^{208} \mathrm{~Pb}$ give much larger value for ${ }^{90} \mathrm{Zr}$, another key nucleus, as compared to the experimental data [14. The GMR energy of ${ }^{90} \mathrm{Zr}$ [15] has since been overestimated by $\sim 1-2 \mathrm{MeV}$ in nonrelativistic [16] and relativistic [17] RPA approach. In the same vain, the GMR energies of Sn and Sm isotopes could not be described within the RPA sum-rule approach [18,19] using the force SkM* with $K_{\infty} \sim 216 \mathrm{MeV}$, which described ${ }^{208} \mathrm{~Pb}$ successfully though. It has been pointed out recently that 
the GMR energies of Sn isotopes are overestimated in a self-consistent RPA approach by $1-2 \mathrm{MeV}$ by the forces which reproduce the GMR energy of ${ }^{208} \mathrm{~Pb}$ appropriately [20,21].

Using the RMF approach and relativistic RPA, a similar behaviour has been observed [22]. The success of the RPA approach and extraction of $K_{\infty}$ using the interpolation method thus hinges very strongly on the nucleus ${ }^{208} \mathrm{~Pb}$ which masks attendant problems with other nuclei.

With the advent of the relativistic mean-field (RMF) theory [23,24,25],26], properties of nuclei and nuclear matter can be studied within this framework which has become successful in describing ground-state properties of nuclei along and far from the stability line [27, 28,29]. The inherent advantage of the RMF theory lies in the Dirac-Lorentz structure of nucleons, which allows a built-in spin-orbit interaction. Consequently, in properties related to shell structure, especially that of the anomalous behaviour of the isotope shifts of $\mathrm{Pb}$ nuclei, the RMF theory exhibits special advantages over the nonrelativistic Skyrme theory [30].

The breathing-mode isoscalar GMR has been studied within the RMF theory earlier [31]. Again, the theoretical or microscopic method of extracting $K_{\infty}$ entails an interpolation amongst various forces with different values of $K_{\infty}$ with a view to being able to reproduce GMR energies of nuclei such as ${ }^{208} \mathrm{~Pb}$, Sn isotopes and ${ }^{90} \mathrm{Zr}$ among others within a microscopic approach [32]. The Lagrangian model with nonlinear scalar self-couplings of $\sigma$ meson has mostly been used with this approach. It has been pointed out in a schematic approach that forces with $K_{\infty} \sim 250-270$ in the RMF model with the nonlinear scalar self-couplings (of the form $\sigma^{3}+\sigma^{4}$ ) would be compatible with the experimental GMR energy of ${ }^{208} \mathrm{~Pb}$ [32]. However, it has been shown recently that the GMR data on Sn isotopes and ${ }^{90} \mathrm{Zr}$ were overestimated by such a force [21. Relativistic RPA calculations have also been performed with Lagrangians with density-dependent meson couplings [33], which focus only on the key nucleus of ${ }^{208} \mathrm{~Pb}$.

The interpolation scheme has been the cornerstone of the theoretical method to extract the incompressibility of nuclear matter microscopically. Using the various Lagrangian models developed, it will be shown in this work that this approach runs into problems due to a sensitivity shown by GMR energies to the Lagrangian model employed. A difference in the density dependence of the meson masses amongst the various Lagrangian models and an ensuing difference in the response of the surface of nuclei to compression is ascribed for this behaviour.

The subject matter of the paper is organized as follows: Definitions of the basic ingradients of the GMR and the incompressibility of the nuclear matter are 
given in Section 2. In Section 3, the formalism of the RMF theory is provided. We discuss the Lagrangian models used in this work in Section 4. Details of the generator coordinate method employed to explore and calculate the GMR energies of nuclei are discussed in Section 5. It will be shown in Section 6 that the GMR energies exhibit a sensitivity to the Lagrangian model considered. We shall show that the surface incompressibility plays an important role for the GMR energies in the RMF theory. The last section summarizes the main results and an outlook for future investigations is presented.

\section{The breathing-mode giant monopole resonance and the com- pressibility of nuclear matter}

The isoscalar $(\mathrm{T}=0)$ giant monopole resonance (GMR) has been well established in the last few decades [6]. It is a breathing-mode of a nucleus wherein the nucleus undergoes a density oscillation about its equilibrium value. The breathing mode GMR energy or its frequency is related to the incompressibility $K_{A}$ of a nucleus with mass number $A$ by

$$
E_{0}=\hbar \sqrt{\frac{K_{A}}{m\left\langle r^{2}\right\rangle}},
$$

where $m$ is the nucleon mass and $\left\langle r^{2}\right\rangle$ is the mean-square mass radius of the nucleus in its ground state.

Analogous to the expansion of the total energy of a nucleus in terms of a liquid drop formula, one can write the expansion of the incompressibility of the nucleus in terms of contributions from the volume, surface, asymmetry, Coulomb and curvature terms, respectively, as [4]:

$$
\begin{aligned}
K_{A} & =\sum K_{i} c_{i} \\
& =K_{\infty}+K_{\text {surf }} A^{-1 / 3}+K_{\text {asym }}\left(\frac{N-Z}{A}\right)^{2}+K_{\text {Coul }} \frac{Z^{2}}{A^{4 / 3}}+K_{\text {curv }} A^{-2 / 3}
\end{aligned}
$$

where $N$ and $Z$ are the neutron and proton numbers, respectively. The $K_{i}{ }^{\prime}$ s are 'incompressibilities' (coefficients) representing various terms and $c_{i}$ 's are the terms of the expansion in various powers of $A$. The volume term $K_{\infty}$ is defined as the curvature of the EOS of the infinite nuclear matter:

$$
K_{\infty}=\left.9 \rho_{0}^{2} \frac{d^{2} E / A}{d \rho^{2}}\right|_{\rho_{0}}
$$


where the second-derivative of the EOS, i.e., of $\epsilon(\rho)$ is evaluated at the saturation density $\rho_{0}$. Thus, $K_{\infty}$ represents the most significant (reference) point of the high-density behaviour of the infinite nuclear matter.

The volume and surface terms provide the most significant contributions to $K_{A}$, whereas the asymmetry and the Coulomb terms are much smaller in comparison. The curvature term is even smaller than the latter two. The volume term being the largest contributor to $K_{A}$ influences the breathingmode GMR energies of nuclei directly.

The Coulomb term $K_{\text {Coul }}$ has been derived as [4]

$$
K_{\text {Coul }}=\frac{3}{5} \frac{e^{2}}{r_{0}}\left(1-\left.\frac{27 \rho_{0}^{2}}{K_{\infty}} e^{\prime \prime \prime}\right|_{\rho_{0}}\right)
$$

where $e^{\prime \prime \prime}=d^{3}(E / A) / d \rho^{3}$ is the third-derivative (skewness) of the EOS. As readily seen from Eq. (4), $K_{\text {Coul }}$ is correlated to $K_{\infty}$. In Section 6 we shall see that the magnitude of the coefficient $K_{\text {Coul }}$ is about two orders of magnitude smaller than the major contributors such as $K_{\infty}$ and $K_{\text {surf }}$. Due to this correlation superimposed on to the meagre quantity of the Coulomb term, it is difficult to extract $K_{\infty}$ from breathing-mode energies [13]. In the present work, we shall circumvent this correlation and attempt to disentangle the various terms.

\section{The Relativistic Mean-Field Theory - Formalism}

The starting point of the RMF theory is the basic Lagrangian (the linear Walecka model) that describes nucleons as Dirac spinors interacting with the meson fields [23]:

$$
\begin{aligned}
\mathcal{L}_{0}= & \bar{\psi}\left(\not p-g_{\omega} \psi-g_{\rho} \vec{\phi} \vec{\tau}-\frac{1}{2} e\left(1-\tau_{3}\right) A-g_{\sigma} \sigma-m\right) \psi \\
& +\frac{1}{2} \partial_{\mu} \sigma \partial^{\mu} \sigma-\frac{1}{2} m_{\sigma}^{2} \sigma^{2}-\frac{1}{4} \Omega_{\mu \nu} \Omega^{\mu \nu}+\frac{1}{2} m_{\omega}^{2} \omega_{\mu} \omega^{\mu} \\
& -\frac{1}{4} \vec{R}_{\mu \nu} \vec{R}^{\mu \nu}+\frac{1}{2} m_{\rho}^{2} \vec{\rho}_{\mu} \vec{\rho}^{\mu}-\frac{1}{4} F_{\mu \nu} F^{\mu \nu}
\end{aligned}
$$

where $m$ is the bare nucleon mass and $\psi$ is its Dirac spinor. Nucleons interact with the $\sigma, \omega$, and $\rho$ mesons. Here, $g_{\sigma}, g_{\omega}$, and $g_{\rho}$ are the respective coupling constants of the interaction. The photonic field is produced by the electromagnetic vector $A^{\mu}$. 
The field tensors of the vector mesons and of the electromagnetic field are given by:

$$
\begin{aligned}
& \Omega^{\mu \nu}=\partial^{\mu} \omega^{\nu}-\partial^{\nu} \omega^{\mu} \\
& \mathbf{R}^{\mu \nu}=\partial^{\mu} \boldsymbol{\rho}^{\nu}-\partial^{\nu} \boldsymbol{\rho}^{\mu} \\
& F^{\mu \nu}=\partial^{\mu} \mathbf{A}^{\nu}-\partial^{\nu} \mathbf{A}^{\mu} .
\end{aligned}
$$

The variational principle gives rise to the Dirac equation:

$$
(-i \alpha \cdot \nabla+V(\mathbf{r})+\beta m *) \psi_{i}=\epsilon_{i} \psi_{i}
$$

where $V(\mathbf{r})$ represents the vector potential:

$$
V(\mathbf{r})=g_{\omega} \omega_{0}(\mathbf{r})+g_{\rho} \tau_{3} \rho_{0}(\mathbf{r})+e \frac{1-\tau_{3}}{2} A_{0}(\mathbf{r}),
$$

and $S(\mathbf{r})$ is the scalar potential

$$
S(\mathbf{r})=g_{\sigma} \sigma(\mathbf{r})
$$

The effective mass is defined by the scalar potential as

$$
m^{*}(\mathbf{r})=m+S(\mathbf{r})
$$

The corresponding Klein-Gordon equations can be written as:

$$
\begin{aligned}
\left(-\Delta+m_{\sigma}^{* 2}\right) \sigma & =-g_{\sigma} \bar{\psi} \psi \\
\left(-\Delta+m_{\omega}^{* 2}\right) \omega_{\nu} & =g_{\omega} \bar{\psi} \gamma_{\nu} \psi \\
\left(-\Delta+m_{\rho}^{* 2}\right) \vec{\rho}_{\nu} & =g_{\rho} \bar{\psi} \gamma_{\nu} \vec{\tau} \psi \\
-\Delta A_{\nu} & =\frac{1}{2} e \bar{\psi}\left(1+\tau_{3}\right) \gamma_{\nu} \psi
\end{aligned}
$$

For the linear Lagrangian model,

$$
m_{\sigma}^{*}=m_{\sigma} ; \quad m_{\omega}^{*}=m_{\omega} ; \quad m_{\rho}^{*}=m_{\rho} .
$$

Thus, the meson masses do not exhibit a density dependence in the linear model. For an even-even nucleus with time-reversal symmetry, the spatial 
components of the vector fields $\boldsymbol{\omega}, \boldsymbol{\rho}_{\mathbf{3}}$ and A vanish. The Klein-Gordon equations for the meson fields are then time-independent inhomogeneous equations with the nucleon densities as sources:

$$
\begin{aligned}
\left(-\Delta+m_{\sigma}^{2}\right) \sigma(\mathbf{r}) & =-g_{\sigma} \rho_{s}(\mathbf{r}) \\
\left(-\Delta+m_{\omega}^{2}\right) \omega_{0}(\mathbf{r}) & =g_{\omega} \rho_{v}(\mathbf{r}) \\
\left(-\Delta+m_{\rho}^{2}\right) \rho_{0}(\mathbf{r}) & =g_{\rho} \rho_{3}(\mathbf{r}) \\
-\Delta A_{0}(\mathbf{r}) & =e \rho_{c}(\mathbf{r}) .
\end{aligned}
$$

where the source terms $\rho_{s}, \rho_{v}, \rho_{3}$ and $\rho_{p}$ to the Klein-Gordon equations (Eq. 13) are the scalar, vector, isovector and charge densities, respectively, as defined by nucleon spinors:

$$
\begin{aligned}
\rho_{s} & =\sum_{i=1}^{A} \bar{\psi}_{i} \psi_{i} \\
\rho_{v} & =\sum_{i=1}^{A} \psi_{i}^{+} \psi_{i} \\
\rho_{3} & =\sum_{p=1}^{Z} \psi_{p}^{+} \psi_{p}-\sum_{n=1}^{N} \psi_{n}^{+} \psi_{n} \\
\rho_{c} & =\sum_{p=1}^{Z} \psi_{p}^{+} \psi_{p} .
\end{aligned}
$$

Here, the sums are taken over the valence nucleons only. The stationary state solutions $\psi_{i}$ are obtained from the coupled system of Dirac (Eq. 7) and KleinGordon equations (Eq. 13) self-consistently.

The total ground-state energy of a spherical nucleus can be expressed as a functional of the baryon spinors $\left\{\psi_{i}\right\}$

$$
E_{R M F}[\psi] \equiv\langle\Phi|\hat{H}| \Phi\rangle
$$

obtained using the Hamiltonian density

$$
\begin{aligned}
\mathcal{H}_{R M F}(r)= & \tau(r)+m \rho_{s}(r) \\
& +\frac{1}{2} g_{\sigma} \rho_{s}(r) \sigma(r)+\frac{1}{2} g_{\omega} \rho_{v}(r) \omega^{0}(r) \\
& +\frac{1}{2} g_{\rho} \rho_{3}(r) \rho^{0}(r)+\frac{1}{2} e \rho_{p}(r) A^{0}(r) \\
& +\frac{1}{2}\left((\nabla \sigma(r))^{2}+m_{\sigma}^{2} \sigma^{2}(r)\right. \\
& -\frac{1}{2}\left(\left(\nabla \omega^{0}(r)\right)^{2}+m_{\omega}^{2}\left(\omega^{0}(r)\right)^{2}\right.
\end{aligned}
$$




$$
\begin{aligned}
& -\frac{1}{2}\left(\left(\nabla \rho^{0}(r)\right)^{2}+m_{\rho}^{2}\left(\rho^{0}(r)\right)^{2}\right. \\
& -\frac{1}{2}\left(\left(\nabla A^{0}(r)\right)^{2}\right.
\end{aligned}
$$

which is given in terms of the source densities (Eq. 14). The kinetic energy density is given by

$$
\tau(r) \equiv \sum_{i=1}^{A} \psi_{i}^{\dagger}(r)\{-i \alpha \nabla\} \psi_{i}(r) .
$$

Taking the variation of Eq. (15) with respect to $\psi_{i}^{\dagger}$, the stationary Dirac equation (Eq. 7) with energy eigenvalues $\epsilon_{i}$ is obtained:

$$
\hat{h}_{D} \psi_{i}(r)=\epsilon_{i} \psi_{i}(r)
$$

where

$$
\hat{h}_{D}=-i \alpha \nabla+\beta m^{*}+g_{\omega} \omega^{0}(r)+g_{\rho} \tau_{3} \rho^{0}(r)+e \frac{\left(1-\tau_{3}\right)}{2} A^{0}(r) .
$$

Solving the Dirac equation (Eq. 18) self-consistently, the ground-state $\Phi_{0}$ of the nucleus is written as a Slater determinant of single-particle spinors $\psi_{i}$ (i $=1,2, \ldots, \mathrm{A})$.

The linear Walecka model has been successful in attaining saturation of nuclear matter as a delicate balance between large fields due to $\sigma$ and $\omega$ mesons. However, a proper description of the properties of finite nuclei was not possible until nonlinear self-couplings of the $\sigma$ meson were introduced [34].

\section{The RMF Lagrangian models}

In this work, we have considered three successful RMF Lagrangian models in order to analyze the breathing-mode isoscalar GMR. In the following, we provide a brief description of the formulation of the various Lagrangian models considered.

\subsection{The nonlinear- $\sigma$ model}

The nonlinear sigma (NL $\sigma$ ) model is the standard Lagrangian model that is used most commonly for calculation of the ground-state properties of nuclei. 
An important ingredient of the NL $\sigma$ model is the assumption of nonlinear scalar self-couplings of the form [34]:

$$
U_{N L}=\frac{1}{3} g_{2} \sigma^{3}+\frac{1}{4} g_{3} \sigma^{4}
$$

The parameters $g_{2}$ and $g_{3}$ are the nonlinear couplings of the $\sigma$-meson in the conventional $\sigma^{3}+\sigma^{4}$ model. The effective Lagrangian for the NL $\sigma$ model becomes

$$
\mathcal{L}_{e f f}=\mathcal{L}_{0}-U_{N L}
$$

The Klein-Gordon equations (Eq. 11) produce a density dependence of the meson masses as given by

$$
\begin{aligned}
& m_{\sigma}^{* 2}=m_{\sigma}^{2}+g_{2} \sigma+g_{3} \sigma^{2} \\
& m_{\omega}^{*}=m_{\omega} \\
& m_{\rho}^{*}=m_{\rho} .
\end{aligned}
$$

Only the $\sigma$-meson mass exhibits an implicit density dependence in the NL $\sigma$ model.

The scalar self-couplings have proved to be important for an appropriate description of nuclear surface and have thus become indispensable. However, the negative quartic coupling in the model NL $\sigma$ has been a source of instability in nuclear matter at higher densities [24,35,36]. It has been shown recently [37] that a scalar-vector interaction (SVI) model that comprises a combination of couplings of $\sigma$ and $\omega$ mesons can dispense with the $\sigma^{3}+\sigma^{4}$ terms in the RMF Lagrangian. The SVI model is able to provide an improved description of the ground-state binding energies and charge radii of nuclei. We shall discuss the basic features of the SVI model in this section below.

The NL $\sigma$ model is well-established and has shown to be a successful model for calculating ground-state properties of nuclei. The earliest parameter sets obtained were $\operatorname{NL1}\left(K_{\infty}=211 \mathrm{MeV}\right)$ [24] and NL2 $\left(K_{\infty}=399 \mathrm{MeV}\right)$ [38]. However, due to the large asymmetry energy $J$, these sets were not appropriate for nuclei away from the stability line. In remedying the problem of a large neutron skin [39], the force NL-SH [28] was developed as one of the first successful parameter sets in the RMF theory, which also described nuclei away from the stability line. Due to a relatively larger value of $K_{\infty}$ of NL-SH, the force NL3 has been obtained [29] with a view to getting a description of the breathing-mode GMR in a physically acceptable region. 
Table 1

The nuclear matter properties of various parameter sets in the NL $\sigma$ model.

\begin{tabular}{lccccc}
\hline Sets & $K_{\infty}(\mathrm{MeV})$ & $E / A(\mathrm{MeV})$ & $m^{*}$ & $\rho_{0}\left(\mathrm{fm}^{-3}\right)$ & $J(\mathrm{MeV})$ \\
\hline NL1 & 211 & -16.42 & 0.573 & 0.152 & 43.5 \\
NL3 & 272 & -16.25 & 0.595 & 0.148 & 37.4 \\
NL-SH & 355 & -16.32 & 0.597 & 0.146 & 36.1 \\
NL2 & 399 & -17.02 & 0.667 & 0.146 & 45.1 \\
\hline
\end{tabular}

In this work, we consider the forces NL1, NL3, NL-SH and NL2, whose order reflects an increasing value of $K_{\infty}$. Nuclear matter properties of these parameter sets are provided in Table 1. It may be noted that even though the forces NL1 and NL2 are quite different in some of the properties such as incompressibility and asymmetry energy, these are able to reproduce ground-state properties of nuclei along the stability line quite well. Here, we have included NL2 in order to extend the systematic behaviour of the NL $\sigma$ model into the region of high incompressibility.

\subsection{The $\sigma-\omega$ coupling model - SIGO}

The $\sigma$ - $\omega$ coupling model (SIGO) has recently been introduced by Haidari and Sharma [40] with a view to bring about an improvement in the ground-state properties of nuclei. A coupling between $\sigma$ and $\omega$ mesons of the form

$$
U_{\sigma \omega}=\frac{1}{2} g_{\sigma \omega} \sigma^{2} \omega^{2}
$$

was introduced in addition to the usual NL $\sigma$ scalar potential of the form $\sigma^{3}$ $+\sigma^{4}$. The effective Lagrangian for the model SIGO becomes

$$
\mathcal{L}_{e f f}=\mathcal{L}_{0}-U_{N L}+U_{\sigma \omega}
$$

The meson mass terms in the corresponding Klein-Gordon equations (Eq. 11) are then given by:

$$
\begin{aligned}
& m_{\sigma}^{* 2}=m_{\sigma}^{2}+g_{2} \sigma+g_{3} \sigma^{2}-g_{\sigma \omega} \omega_{0}^{2} \\
& m_{\omega}^{* 2}=m_{\omega}^{2}+g_{\sigma \omega} \sigma^{2} \\
& m_{\rho}^{*}=m_{\rho}
\end{aligned}
$$


Thus, in the SIGO model, both the $\sigma$ and $\omega$ meson masses exhibit an implicit density dependence.

It has been shown [40] that the parameter set SIG-OM obtained in the model SIGO is able to provide an excellent description of the ground-state properties such as binding energies and charge radii of nuclei along the stability line as well as far away from it. Especially, a significant improvement in the binding energy of nuclei at the magic numbers has been achieved. Consequently, an excellent description of the total binding energy of Sn isotopes all over the range of the shell from ${ }^{100} \mathrm{Sn}(N=50)$ to ${ }^{132} \mathrm{Sn}(N=82)$ was obtained. Charge radii of nuclei, especially of $\mathrm{Pb}$ isotopes, which are over estimated significantly by NL3, were well reproduced. The ensuing EOS of the nuclear matter with SIG-OM has been shown to be much softer than that given by the parameter sets of the NL $\sigma$ model [40].

In order to investigate the behaviour of the model SIGO for the breathingmode GMR, we have constructed a few parameter sets by extending the range of $K_{\infty}$ below and above that of the set SIG-OM $\left(K_{\infty}=265 \mathrm{MeV}\right)$. The parameter sets SIGO-a, SIGO-b, SIGO-c and SIGO-d have been obtained with $K_{\infty}$ ranging between $241-283 \mathrm{MeV}$. The nuclear matter properties of the parameter sets of the model SIGO are given in Table 2. It may be noted that the sets SIGO-a, SIGO-b, SIGO-c and SIGO-d corresponding to different values of $K_{\infty}$ are able to reproduce the binding energy and charge radii of key nuclei from ${ }^{16} \mathrm{O}$ to ${ }^{208} \mathrm{~Pb}$ well. Qualitatively, the set SIG-OM is considered as the best amongst all the sets of the SIGO model provided in Table 2.

Table 2

The nuclear matter properties of the $\sigma-\omega$ coupling (SIGO) sets.

\begin{tabular}{lccccc}
\hline Sets & $K_{\infty}(\mathrm{MeV})$ & $E / A(\mathrm{MeV})$ & $m^{*}$ & $\rho_{0}\left(\mathrm{fm}^{-3}\right)$ & $J(\mathrm{MeV})$ \\
\hline SIGO-a & 241.1 & -15.90 & 0.621 & 0.149 & 33.0 \\
SIGO-b & 248.4 & -16.00 & 0.622 & 0.150 & 33.6 \\
SIG-OM & 265.2 & -16.30 & 0.622 & 0.149 & 37.0 \\
SIGO-c & 272.8 & -16.33 & 0.623 & 0.149 & 37.7 \\
SIGO-d & 282.5 & -16.34 & 0.620 & 0.148 & 37.5 \\
\hline
\end{tabular}




\subsection{The scalar-vector interaction model - SVI}

The scalar-vector interaction (SVI) model has recently been developed by the author [37] with a view to remove nonlinearities of the mesonic fields in the RMF theory. Self-interactions of $\sigma$ field have been cited as a source of instability in the nuclear matter at higher densities [36]. It has now been demonstrated [37] that by a suitable combination of couplings of $\sigma$ and $\omega$ fields, the self-interactions of the mesonic fields can be dispensed with. It is expected that the introduction of SVI would be consistent with a linear realization of the chiral symmetry in a suitable scheme [41].

The SVI model consists of the meson-meson interactions between $\sigma$ and $\omega$ mesons of the form

$$
U_{m m}=\frac{1}{2} g_{4} \sigma \omega^{2}+\frac{1}{2} g_{5} \sigma^{2} \omega^{2},
$$

where $g_{4}$ and $g_{5}$ are the respective coupling constants for interactions between $\sigma$ and $\omega$ mesons. The effective Lagrangian without self-interactions of the bosonic fields thus becomes

$$
\mathcal{L}_{\text {eff }}=\mathcal{L}_{0}+U_{m m} .
$$

The corresponding Klein-Gordon equations (Eq. 11) have the effective meson masses as given by:

$$
\begin{aligned}
m_{\sigma}^{* 2} & =m_{\sigma}^{2}-g_{4} \omega_{0}^{2} /(2 \sigma)-g_{5} \omega_{0}^{2} \\
m_{\omega}^{* 2} & =m_{\omega}^{2}+g_{4} \sigma+g_{5} \sigma^{2} \\
m_{\rho}^{*} & =m_{\rho} .
\end{aligned}
$$

These equations exhibit an implicit density dependence of the $\sigma$ and $\omega$ meson masses. Both the $\sigma$ and $\omega$ meson masses provide a density dependence that is different from that for the SIGO model (Eq. 25). Thus, the density dependence of $\sigma$ and $\omega$ meson masses, as represented by Eqs. (22), (25) and (28) for the three Lagrangian models NL $\sigma$, SIGO and SVI, respectively, are different from one another. A comprehensive discussion of the differences in the density dependences in various Lagrangian models and their influence on nuclear properties shall be presented elsewhere [43]. It shall be instructive to see as to how these density dependences in the high-density regime would compare with the predictions of the Brown-Rho scaling [44]. 
Table 3

The nuclear matter properties of the scalar-vector interaction (SVI) sets.

\begin{tabular}{lccccc}
\hline Sets & $K_{\infty}(\mathrm{MeV})$ & $E / A(\mathrm{MeV})$ & $m^{*}$ & $\rho_{0}\left(\mathrm{fm}^{-3}\right)$ & $J(\mathrm{MeV})$ \\
\hline SVI-a & 243.5 & -16.30 & 0.615 & 0.150 & 37.4 \\
SVI-b & 253.1 & -16.24 & 0.612 & 0.150 & 36.6 \\
SVI-1 & 263.9 & -16.30 & 0.616 & 0.149 & 37.6 \\
SVI-2 & 271.5 & -16.31 & 0.621 & 0.149 & 37.0 \\
SVI-c & 288.1 & -16.25 & 0.617 & 0.148 & 36.4 \\
\hline
\end{tabular}

The parameters sets SVI-1 $\left(K_{\infty}=264 \mathrm{MeV}\right)$ and SVI-2 $\left(K_{\infty}=272 \mathrm{MeV}\right)$ were obtained in Ref. [37]. These sets have been shown to provide an excellent description of the ground-state energies of nuclei over a large range of the periodic table. This includes nuclei along the stability line as well as far away from it. Description of binding energies and charge radii of nuclei that is achieved with the SVI sets is significantly better than that with the NL $\sigma$ set NL3. Having established the basis of the SVI model, we have constructed a few more parameter sets having $K_{\infty}$ in the range $\sim 240-290 \mathrm{MeV}$ for a systematic analysis of the breathing-mode GMR. The nuclear matter properties of the parameter sets SVI-a, SVI-b, and SVI-c thus constructed in addition to those of SVI-1 and SVI-2 are listed in Table 3. It is worth mentioning that the auxiliary sets SVI-a, SVI-b, SVI-c are also able to provide a reasonably good description of the ground-state properties of nuclei.

\section{The generator coordinate method and the breathing-mode GMR}

The generator coordinate method (GCM) is a powerful tool to study ground and excited states in atoms and nuclei. The GCM has been applied to study effects of correlations on the ground-state properties of nuclei using the densitydependent Skyrme approach [45. In the present work, we employ the GCM in the RMF theory to explore the excited state of the isoscalar GMR in atomic nuclei.

The GCM is based upon a trial $A$-particle wavefunction $\Psi_{G C M}$ in the form of a linear combination of

$$
\Psi_{\mathrm{GCM}}\left(\mathbf{r}_{1}, \mathbf{r}_{2}, \ldots, \mathbf{r}_{\mathbf{A}}\right)=\int \mathcal{F}(q) \Phi\left(\mathbf{r}_{1}, \mathbf{r}_{2}, \ldots, \mathbf{r}_{\mathbf{A}} ; q\right) d q
$$

where the generator function $\Phi(q) \equiv \Phi\left(\mathbf{r}_{\mathbf{1}}, \mathbf{r}_{\mathbf{2}}, \ldots, \mathbf{r}_{\mathbf{A}} ; q\right)$ is a Slater determinant 
$\Phi(q)$ constituted from single-particle spinors $\psi_{i}(\mathbf{r}, q),(i=1,2, \ldots, A)$ as a function of the generator coordinate $q$. The "weight" or the "generator function" $\mathcal{F}(q)$ is determined from variation of the total energy of the system

$$
E[\mathcal{F}]=\frac{\left\langle\Psi_{\mathrm{GCM}}|\hat{H}| \Psi_{\mathrm{GCM}}\right\rangle}{\left\langle\Psi_{\mathrm{GCM}} \mid \Psi_{\mathrm{GCM}}\right\rangle}
$$

with respect to $\mathcal{F}(q)$. This leads to the Hill-Wheeler equation for the weight function:

$$
\int\left[\mathcal{H}\left(r ; q, q^{\prime}\right)-E \mathcal{N}\left(r ; q, q^{\prime}\right)\right] \mathcal{F}\left(q^{\prime}\right) d q^{\prime}=0,
$$

where

$$
\mathcal{H}\left(r ; q, q^{\prime}\right)=\left\langle\Phi(q)|\hat{H}| \Phi\left(q^{\prime}\right)\right\rangle
$$

is the overlap energy-density kernel of the Hamiltonian $\hat{H}$ associated with the RMF Lagrangian, and

$$
\mathcal{N}\left(r ; q, q^{\prime}\right)=\left\langle\Phi(q) \mid \Phi\left(q^{\prime}\right)\right\rangle
$$

is the overlap norm kernel. The overlap energy-density kernel takes the form given in Eq. (16):

$$
\begin{aligned}
\mathcal{H}_{R M F}\left(r ; q, q^{\prime}\right)= & \tau\left(r ; q, q^{\prime}\right)+M \rho_{s}\left(r ; q, q^{\prime}\right) \\
& +\frac{1}{2} g_{\sigma} \rho_{s}\left(r ; q, q^{\prime}\right) \sigma\left(r ; q, q^{\prime}\right)+\frac{1}{2} g_{\omega} \rho_{v}\left(r ; q, q^{\prime}\right) \omega^{0}\left(r ; q, q^{\prime}\right) \\
& +\frac{1}{2} g_{\rho} \rho_{3}\left(r ; q, q^{\prime}\right) \rho^{0}\left(r ; q, q^{\prime}\right)+\frac{1}{2} e \rho_{p}\left(r ; q, q^{\prime}\right) A^{0}(r) \\
& +\frac{1}{2}\left(\left(\nabla \sigma\left(r ; q, q^{\prime}\right)\right)^{2}+m_{\sigma}^{2} \sigma^{2}\left(r ; q, q^{\prime}\right)\right. \\
& -\frac{1}{2}\left(\left(\nabla \omega^{0}\left(r ; q, q^{\prime}\right)\right)^{2}+m_{\omega}^{2}\left(\omega^{0}\left(r ; q, q^{\prime}\right)\right)^{2}\right. \\
& -\frac{1}{2}\left(\left(\nabla \rho^{0}\left(r ; q, q^{\prime}\right)\right)^{2}+m_{\rho}^{2}\left(\rho^{0}\left(r ; q, q^{\prime}\right)\right)^{2}\right. \\
& -\frac{1}{2}\left(\left(\nabla A^{0}\left(r ; q, q^{\prime}\right)\right)^{2}\right. \\
& + \text { other terms. }
\end{aligned}
$$

Here, the 'other terms' refer to the meson-meson interaction terms of various Lagrangian models considered in Section 4, The kinetic energy density can be written in terms of the spinors $\left\{\psi_{i}(\mathrm{r} ; q)\right\}$ as

$$
\tau\left(r ; q, q^{\prime}\right)=\sum N_{j i}^{-1} \psi_{i}^{\dagger}(\mathbf{r} ; q)\{-i \alpha \nabla\} \psi_{j}\left(\mathbf{r} ; q^{\prime}\right)
$$


The source densities appearing in Eq. (34) can be written as

$$
\begin{aligned}
& \rho_{s}\left(r ; q, q^{\prime}\right)=\sum N_{j i}^{-1} \bar{\psi}_{i}(\mathbf{r} ; q) \psi_{j}\left(\mathbf{r} ; q^{\prime}\right), \\
& \rho_{v}\left(r ; q, q^{\prime}\right)=\sum N_{j i}^{-1} \psi_{i}^{\dagger}(\mathbf{r} ; q) \psi_{j}\left(\mathbf{r} ; q^{\prime}\right), \\
& \rho_{3}\left(r ; q, q^{\prime}\right)=\sum N_{j i}^{-1} \psi_{i}^{\dagger}(\mathbf{r} ; q) \tau_{3} \psi_{j}\left(\mathbf{r} ; q^{\prime}\right), \\
& \rho_{p}\left(r ; q, q^{\prime}\right)=\sum N_{j i}^{-1} \psi_{i}^{\dagger}(\mathbf{r} ; q) \frac{\left(1-\tau_{3}\right)}{2} \psi_{j}\left(\mathbf{r} ; q^{\prime}\right) .
\end{aligned}
$$

The overlap matrix elements $N_{i j}\left(q, q^{\prime}\right)$ are calculated using the spinors as

$$
N_{i j}\left(q, q^{\prime}\right)=\int d^{3} r \psi_{i}^{\dagger}(\mathbf{r} ; q) \psi_{j}\left(\mathbf{r} ; q^{\prime}\right)
$$

The determinant of $N_{i j}\left(q, q^{\prime}\right)$ provides the overlap kernel (Eq. 33):

$$
\mathcal{N}\left(q, q^{\prime}\right)=\operatorname{det}\left\{N_{i j}\left(q, q^{\prime}\right)\right\} .
$$

On obtaining the energy and norm overlap kernels using Eqs. (321) and (38), the Hill-Wheeler equation (31) is solved. The solution provides the nuclear ground and the excited states.

In order to obtain the isoscalar GMR energy of nuclei, we perform constrained RMF calculations by solving the Dirac equation

$$
\left(\hat{h}_{D}-q \hat{Q}\right) \psi_{i}(x)=\epsilon_{i} \psi_{i}(x)
$$

with the constraint operator $\hat{Q}=r^{2}$. For the case of the isoscalar GMR, the Lagrange multiplier $q$ is associated with the value of the nuclear root-meansquare $(r m s)$ radius

$$
R=\langle\hat{Q}\rangle=\left\{\frac{1}{A} \int r^{2} \rho_{v}(r ; q) d^{3} r\right\}^{1 / 2}
$$

where $\rho_{v}(r ; q)$ is the baryon density determined by the solution $\left\{\psi_{i}(\mathbf{r} ; q)\right\}$ for a given value of the generator coordinate $q$.

Constrained RMF calculations have been performed in coordinate space for a range of the Lagrange multiplier $q$. Constructing the generator Slater determinant $\Phi(q)$, the integral kernels, Eq. (32) and Eq. (33) are obtained. On solving the Hill-Wheeler equation, the first excited state gives the energy of the isoscalar GMR. Details of the GCM method applied to the RMF theory have been provided in Ref. [31]. 


\section{Results and discussion}

As noted earlier in Section 4, the Lagrangian sets SIG-OM in the SIGO model [40] and SVI-1 and SVI-2 in the SVI model [37] developed recently have been shown to bring about a significant improvement in the ground-state properties of nuclei as compared to the parameter set NL3 of the NL $\sigma$ model. With the advent of the alternative Lagrangian models SIGO and SVI, we have now explored the response of various Lagrangian models to the breathing-mode isoscalar GMR.

As alluded to in Ref. [40], the set SIG-OM yields GMR energies which are larger than those of NL3, though the incompressibility $K_{\infty}$ of SIG-OM is smaller than that of NL3. It seems paradoxical, for according to conventional wisdom, a force with a higher value of $K_{\infty}$ should yield a higher value of the GMR energy and vice versa. This strange feature has prompted us to investigate the GMR response within the framework of the RMF theory employing various Lagrangian models at hand. The isoscalar GMR energies have been calculated for a few key nuclei using the GCM approach.

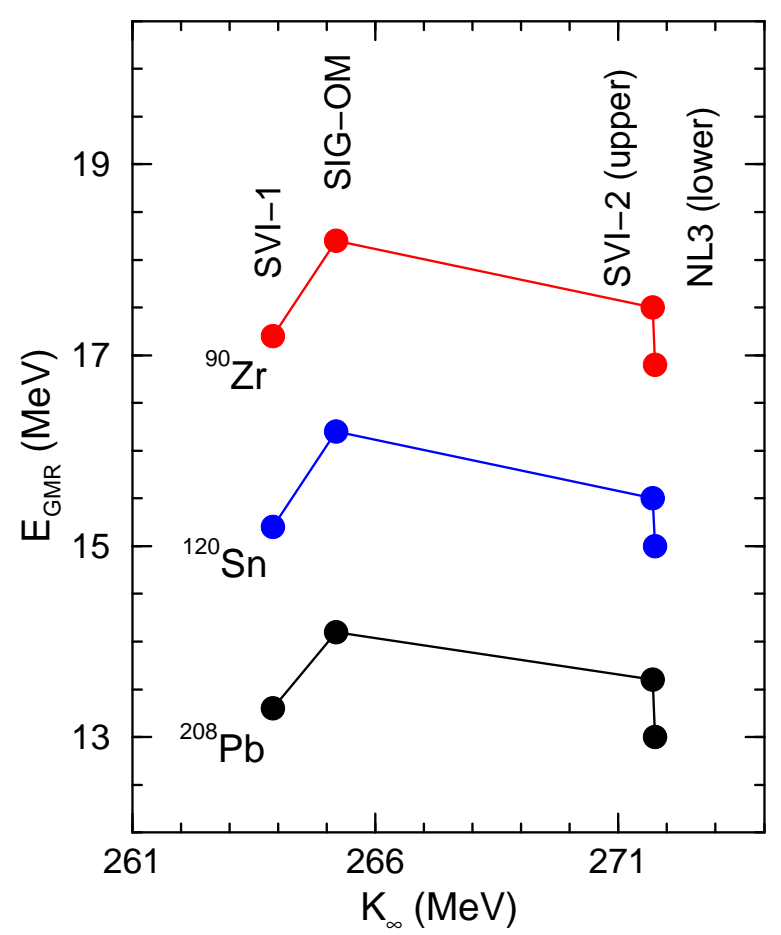

Fig. 1. The breathing-mode GMR energies for nuclei ${ }^{208} \mathrm{~Pb},{ }^{120} \mathrm{Sn}$ and ${ }^{90} \mathrm{Zr}$ obtained with the generator coordinate method using the NL $\sigma$ Lagrangian set NL3, the SVI Lagrangian sets SVI-1 and SVI-2 and the SIGO Lagrangian set SIG-OM. The sets NL3 and SVI-2 have nearly the same value of $K_{\infty}$. 


\subsection{The GMR energies: a paradoxical picture}

In order to visualize the response of various Lagrangian models, we show the GMR energies obtained by employing the GCM approach in Fig. 1, The GMR energies for three key nuclei of ${ }^{208} \mathrm{~Pb},{ }^{120} \mathrm{Sn}$ and ${ }^{90} \mathrm{Zr}$ have been obtained using the parameter set NL3 of the NL $\sigma$ model, with the sets SVI-1 and SVI-2 of the SVI model [37] and with the set SIG-OM of the SIGO model [40]. Contrary to the usual increase of the GMR energy with $K_{\infty}$, an unusual pattern displaying rather paradoxical behaviour is seen. The GMR energies obtained with SIGOM are bigger than those of NL3 as mentioned above. These are also bigger than those of SVI-2. Moreover, SVI-2 values are bigger than those of NL3, though SVI-2 and NL3 have nearly same value of $K_{\infty}$. This behaviour implies that some finite-size contribution(s) to the incompressibility must be different in these Lagrangian models. Such a behaviour was noted in relativistic RPA calculations in Ref. [46]. It was shown that the force NL-SH of NL $\sigma$ model and TM1 with quartic $\omega$ coupling [47], both having very different values of $K_{\infty}$, were shown to provide comparable values of the GMR energy for ${ }^{208} \mathrm{~Pb}$. In order to throw light upon the seemingly paradoxical behaviour of the GMR energies and with a view to gauge the response of the finite-size effects to the nuclear incompressibility of finite nuclei, we have first analyzed the GMR energies within each Lagrangian model separately.

\subsection{GMR energies with the NLo model}

In the NL $\sigma$ model, we have employed the standard parameter sets NL1, NL3, NL-SH and NL2, which are in an increasing sequence of $K_{\infty}$. These span a large range of $K_{\infty} \sim 210-400 \mathrm{MeV}$. The isoscalar $(\mathrm{T}=0)$ GMR energies calculated for nuclei ${ }^{208} \mathrm{~Pb},{ }^{120} \mathrm{Sn}$ and ${ }^{90} \mathrm{Zr}$ using these parameter sets are shown in Fig. 2. The GMR energies are shown directly as a function of $K_{\infty}$ in Fig. 2(a). Due to obvious dependence of $E_{G M R}$ on $K_{A}$, the curves exhibit a quadratic like dependence on $K_{\infty}$. Only for ${ }^{90} \mathrm{Zr}$ does the energy seems to fall slightly out of the trend especially for NL2. This may be due to an extremely large value of $K_{\infty} \sim 400 \mathrm{MeV}$ for NL2, whereby some anharmonicity may creep in for ${ }^{90} \mathrm{Zr}$, which is relatively light as compared to ${ }^{208} \mathrm{~Pb}$. It is interesting to note that the curve for each nucleus and especially that for the heavy nucleus ${ }^{208} \mathrm{~Pb}$ shows a monotonic dependence on $K_{\infty}$, though each of the parameter sets was constructed under different circumstances. Some nuclear matter properties of these sets such as the asymmetry energy are known to be rather different especially that of NL1 and NL2 with a value of $J \sim 44 \mathrm{MeV}$.

The GMR energies are shown as a function of $\sqrt{K_{\infty}}$ in Fig. 2(b) in order to

eliminate the apparent quadratic dependence. The curve for ${ }^{208} \mathrm{~Pb}$ shows a 


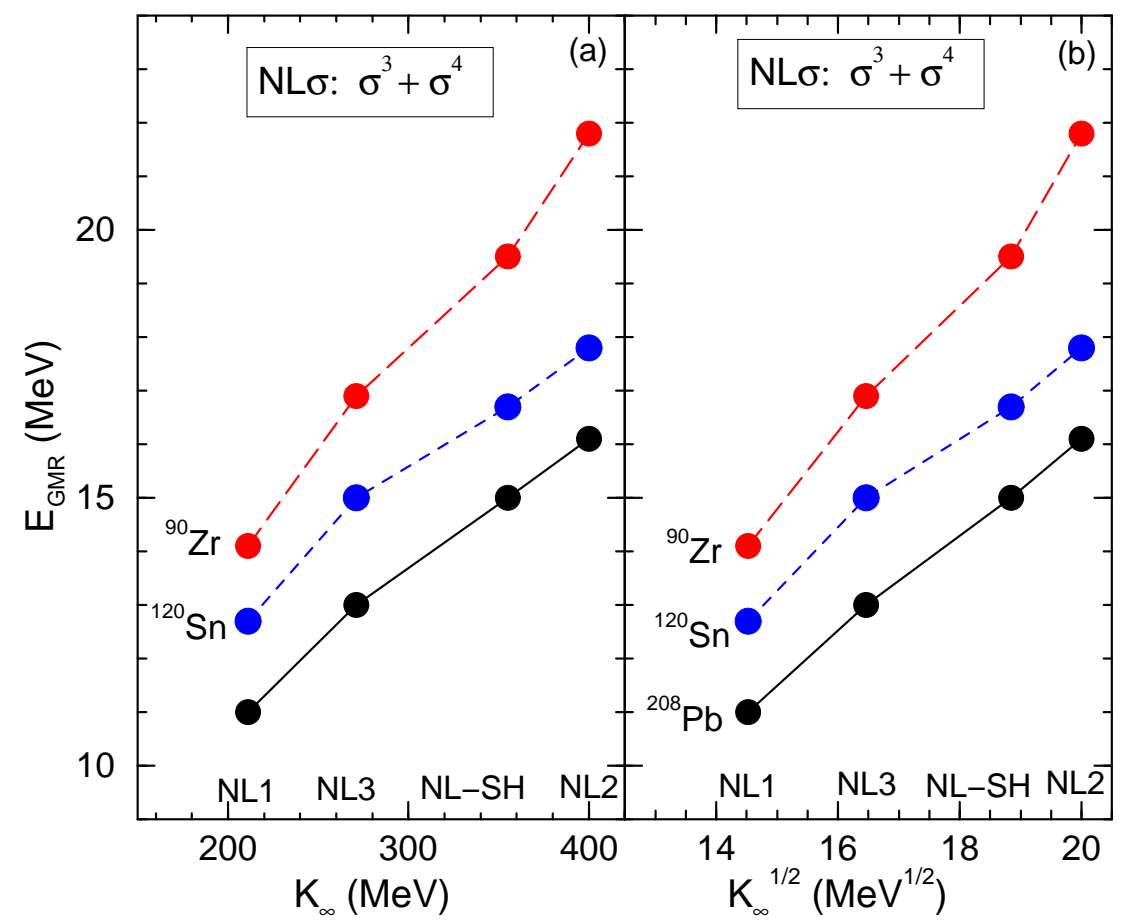

Fig. 2. The breathing-mode isoscalar GMR energies for ${ }^{208} \mathrm{~Pb},{ }^{120} \mathrm{Sn}$ and ${ }^{90} \mathrm{Zr}$ obtained with the standard parameter sets NL1, NL3, NL-SH and NL2 of the NL $\sigma$ model in an increasing sequence of $K_{\infty}$. The energies $E_{G M R}$ are displayed as a function of (a) $K_{\infty}$ and (b) $\sqrt{K_{\infty}}$.

slight improvement towards a linear behaviour. For ${ }^{120} \mathrm{Sn}$ and ${ }^{90} \mathrm{Zr}$ a similar feature may not be visible due to interplay of stronger finite-size effects such as the surface which becomes important for medium heavy and lighter nuclei.

\subsection{The GMR energies with the SVI model}

We have calculated the breathing-mode GMR energies within the scalar-vector Lagrangian model SVI using the GCM with the parameter sets SVI-a, SVI-b, SVI-1, SVI-2 and SVI-c, which are in an increasing order of $K_{\infty}$. These sets encompass a region of $K_{\infty} \sim 240-290 \mathrm{MeV}$. The results are shown in Fig. 3 . The curves show a monotonous behaviour with $K_{\infty}$. All the parameter sets of SVI fall in an orderly pattern and show a dependence on $K_{\infty}$ similar to that seen in Fig. 2. However, as compared to the values in Fig. 2, the GMR energies with SVI are higher than the corresponding values with the NL $\sigma$ model. 


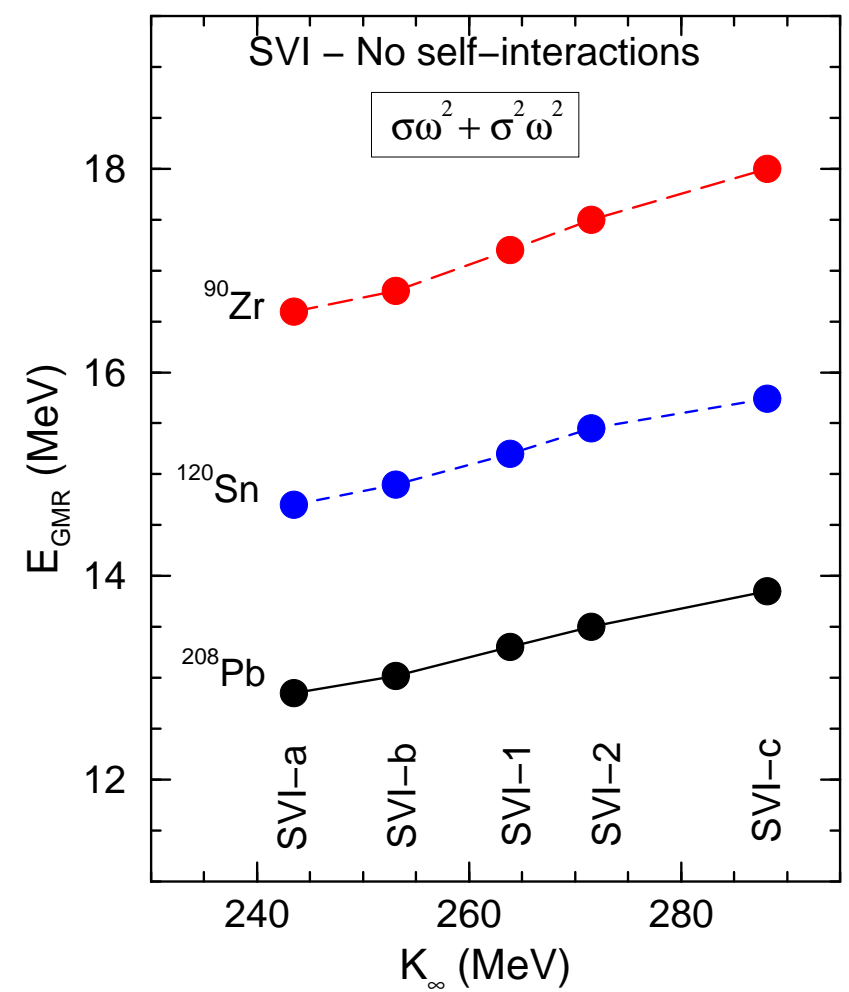

Fig. 3. The breathing-mode isoscalar GMR energies for ${ }^{208} \mathrm{~Pb},{ }^{120} \mathrm{Sn}$ and ${ }^{90} \mathrm{Zr}$ obtained with the parameter sets SVI-a, SVI-b, SVI-1, SVI-2, and SVI-c of the scalar-vector interaction model SVI of Ref. [37.

\subsection{The GMR energies with the SIGO model}

We have then investigated the response of the $\sigma-\omega$ model SIGO to the breathingmode GMR. GCM calculations have been performed with the parameter sets SIGO-a, SIGO-b, SIG-OM, SIGO-c and SIGO-d. The results are shown in Fig. 4. All the SIGO sets display an orderly pattern such as that seen in Figs. 2 and 3 with the NL $\sigma$ and SVI models, respectively. Comparatively, the SIGO values are higher than the corresponding SVI values.

\subsection{A comparative picture}

The results obtained with the three Lagrangian models are compared in Fig. 5. Focusing on the region of physically acceptable values of $K_{\infty}$, the figure shows the GMR energies for the three Lagrangian models within the range of $K_{\infty} \sim$ $230-320 \mathrm{MeV}$. For a given value of $K_{\infty}$, the model NL $\sigma$ gives the lowest values, whereas the model SIGO delivers the largest values for the breathing-mode GMR energy. Thus, the models NL $\sigma$, SVI and SIGO, respectively, produce 


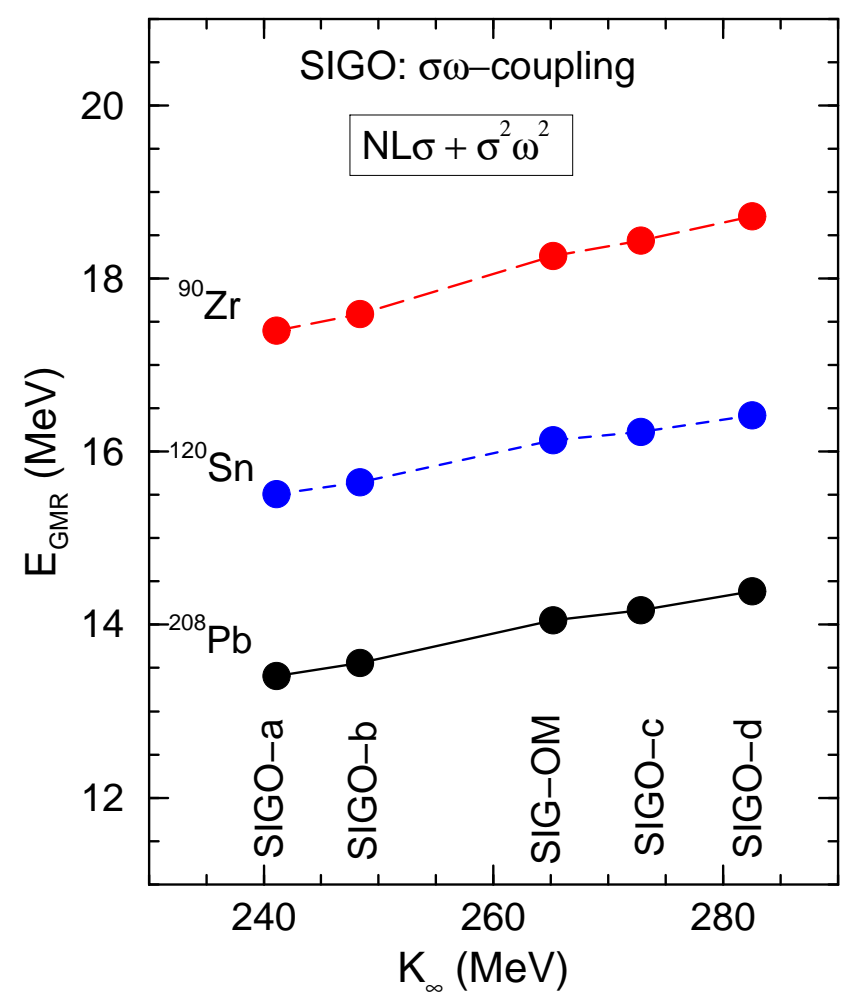

Fig. 4. The GMR energies for ${ }^{208} \mathrm{~Pb},{ }^{120} \mathrm{Sn}$ and ${ }^{90} \mathrm{Zr}$ obtained with the parameter sets SIGO-a, SIGO-b, SIG-OM, SIGO-c and SIGO-d of the $\sigma-\omega$ model SIGO of Ref. [40]

GMR energies in an increasing order for any given nucleus. The results in this figure demonstrate that the GMR energies depend strongly upon the Lagrangian model employed and that each model predicts a different value of the GMR energy for a given $K_{\infty}$. This implies that the GMR energy is not a simple function of only the incompressibility $K_{\infty}$ of the infinite nuclear matter. Different values of the GMR energy for a given value of $K_{\infty}$ indicate different contribution of finite-size effect(s) to the incompressibility $K_{A}$ of a nucleus. We discuss below the relative magnitudes of various contributions to $K_{A}$ and attempt to discern the factors responsible for multiplicity of the GMR energies for a given value of $K_{\infty}$ and thus the reason for producing the paradoxical behaviour seen in Fig. 1.

The multiplicity of the GMR energies for a given $K_{\infty}$ in the RMF theory is in contrast to a single value that is obtained usually within the density-dependent Skyrme theory [14]. This can be attributed to a difference in the density dependence of the nuclear interaction amongst the various Lagrangian models. In comparison, the density dependence within the non-relativistic Skyrme approach is provided by the standard Skyrme density functional used universally - which is fixed at the outset. Should an alternative form of the density functional in the Skyrme approach arise, a situation akin to the RMF theory would 
emerge. Microscopic differences amongst various Lagrangian models, which lead to a phenomenal difference in the GMR energies, are being investigated separately [43].

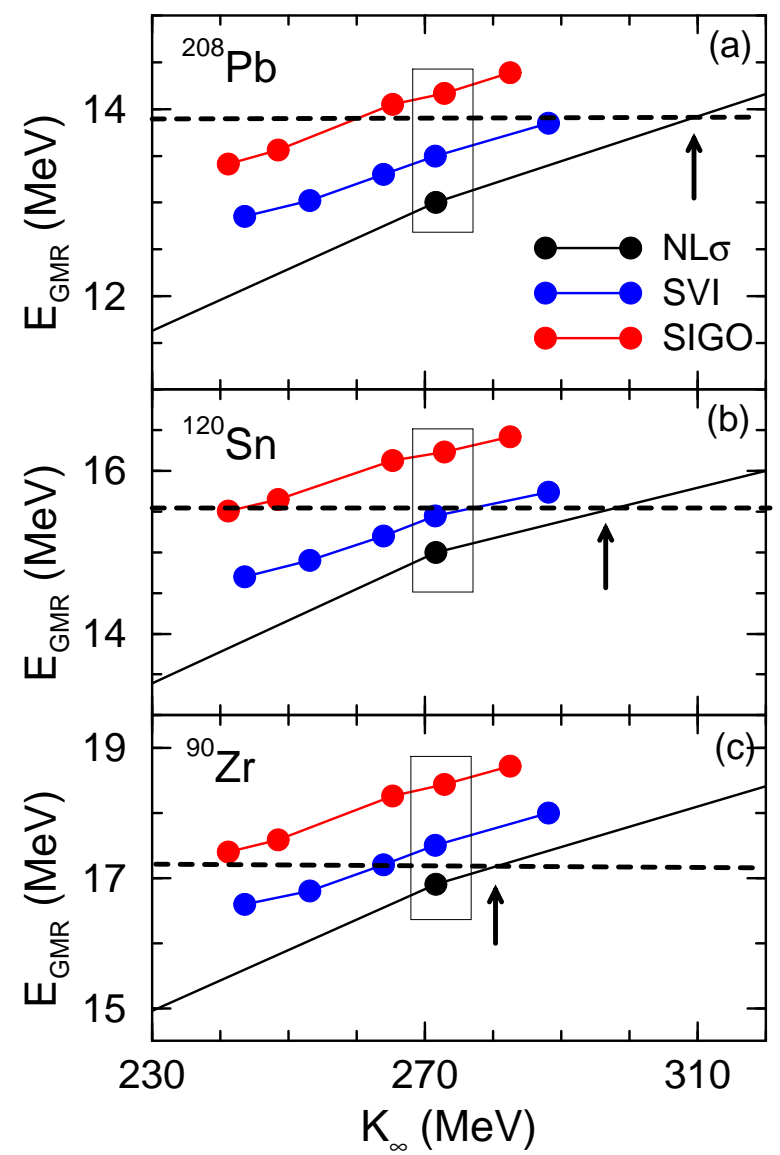

Fig. 5. The GMR energies for ${ }^{208} \mathrm{~Pb},{ }^{120} \mathrm{Sn}$ and ${ }^{90} \mathrm{Zr}$ for the three Lagrangian models in the physically acceptable region of $K_{\infty}$ compared with the experimental data. The arrows show intersection of the experimental data (dashed lines) with the theoretical curves obtained with the NL $\sigma$ model. The results with sets having nearly the same value of $K_{\infty} \sim 272 \mathrm{MeV}$ in the three Lagrangian models are enclosed by rectangular boxes.

\subsection{Theoretical extraction of $K_{\infty}-$ a conundrum}

Given the emerging situation in the RMF theory, an intersection of the experimental values (shown by the dashed horizontal lines in Fig. 5) with the theoretical curves would yield a different value of $K_{\infty}$ depending upon the Lagrangian model used. The experimental values shown by the dashed lines cross the curves at different locations in $K_{\infty}$. Staying with the pivotal case of ${ }^{208} \mathrm{~Pb}$ in Fig. 5(a), each Lagrangian model yields $K_{\infty}$ value which is different 
from the others. $K_{\infty}$ inferred from the datum of ${ }^{208} \mathrm{~Pb}$ with the model $\mathrm{NL} \sigma$ is $\sim 310 \mathrm{MeV}$, whereas a value of $\sim 290 \mathrm{MeV}$ and $\sim 260 \mathrm{MeV}$ would be determined with the models SVI and SIGO, respectively.

Using the datum on ${ }^{120} \mathrm{Sn}$ in Fig. 馬(b), the value of $K_{\infty}$ inferred is $\sim 300 \mathrm{MeV}$, $\sim 275 \mathrm{MeV}$ and $\sim 245 \mathrm{MeV}$ with the NL $\sigma$, SVI and SIGO models, respectively. Similarly, using the datum on ${ }^{90} \mathrm{Zr}$ in Fig. [5(c), one would conclude a value of $K_{\infty}$ as $\sim 280 \mathrm{MeV}, \sim 265 \mathrm{MeV}$ and $\sim 240 \mathrm{MeV}$, respectively. The shift in the value of $K_{\infty}$ inferred from a given model using the experimental data on ${ }^{208} \mathrm{~Pb},{ }^{120} \mathrm{Sn}$ and ${ }^{90} \mathrm{Zr}$ is illustrated by the arrows for the case of the NL $\sigma$ model. A similar shift is evident in the figure (not shown by arrows) for the models SVI and SIGO as well.

Staying with the interpolation method, it is equally illustrative to conclude that any of the Lagrangian models is not able to match the experimental data on all the three nuclei simultaneously with a given value of $K_{\infty}$. These observations bring into question the hitherto used theoretical approach based upon interpolation amongst forces. Thus, the approach of determining $K_{\infty}$ á la Blaizot [4] becomes unsustainable in the RMF theory. It is therefore essential to 'calibrate' the density dependence of the nuclear interaction in the RMF theory before such an approach could be applicable. Arguably, the form of the density dependence opens up another dimension in the landscape of the breathing-mode GMR and the incompressibility of nuclear matter.

The shift of the arrows from the right towards the left in going from ${ }^{208} \mathrm{~Pb}$ to ${ }^{90} \mathrm{Zr}$ (i.e., a decrease in the value of $K_{\infty}$ so inferred) needs analysis. It is understood that the interior (or bulk) of the nucleus ${ }^{208} \mathrm{~Pb}$ has a density close to that of the saturation density of nuclear matter and hence is synonymous with the nuclear matter for practical purposes. It has a nuclear surface that is smaller as compared to that in ${ }^{120} \mathrm{Sn}$ and ${ }^{90} \mathrm{Zr}$. Thus, in going from ${ }^{208} \mathrm{~Pb}$ to ${ }^{90} \mathrm{Zr}$, the region of the nuclear surface increases. The effect of compression of a correspondingly large nuclear surface needs to be accounted for. The continuous shift in the arrows (and hence $K_{\infty}$ value inferred) from ${ }^{208} \mathrm{~Pb}$ to ${ }^{90} \mathrm{Zr}$ points towards the increased role played by an increasingly larger surface region.

\subsection{Disentangling the finite-size effects and the surface incompressibility}

A shift in the value of $K_{\infty}$ extracted from the experimental GMR energies of ${ }^{208} \mathrm{~Pb},{ }^{120} \mathrm{Sn}$ and ${ }^{90} \mathrm{Zr}$, as shown in Fig. 囵, renders the approach of interpolation amongst forces as futile. It is, therefore, pertinent to revisit the expansion of $K_{A}$ to dissect various components. As noted earlier, the use of Eq. (2) does not allow an unambiguous determination of $K_{\infty}$ primarily due to the correlation of 
the Coulomb term to $K_{\infty}$ superimposed on to a much smaller magnitude of the former. Here, we adopt a slightly different approach. Instead of allowing the aforesaid correlation to jeopardise the extraction of $K_{\infty}$, we let the coefficient $K_{\text {Coul }}$ fit as an independent variable.

For a comparative analysis of the GMR energies, we have selected the parameters sets having the same value of $K_{\infty}$ in the three different Lagrangian models. The sets with an incompressibility close to $K_{\infty} \sim 272 \mathrm{MeV}$ are enclosed by the rectangular boxes in Fig. 5. This corresponds to the sets NL3 $\left(K_{\infty}=271.8 \mathrm{MeV}\right)$, SVI-2 $\left(K_{\infty}=271.4 \mathrm{MeV}\right)$ and SIGO-c $\left(K_{\infty}=272.8\right.$ $\mathrm{MeV}$ ) in the Lagrangian models NL $\sigma$, SVI and SIGO, respectively.

Table 4

The GMR energies for ${ }^{208} \mathrm{~Pb},{ }^{90} \mathrm{Zr},{ }^{48} \mathrm{Ca}$, and ${ }^{40} \mathrm{Ca}$ obtained with the parameter sets NL3, SVI-2 and SIGO-c having the same value of $K_{\infty} \sim 272 \mathrm{MeV}$ in the three Lagrangian models.

\begin{tabular}{cccc}
\hline Nucleus & NL3 & SVI-2 & SIGO-c \\
\hline${ }^{208} \mathrm{~Pb}$ & 13.0 & 13.5 & 14.2 \\
${ }^{90} \mathrm{Zr}$ & 16.9 & 17.5 & 18.4 \\
${ }^{48} \mathrm{Ca}$ & 18.9 & 19.7 & 20.5 \\
${ }^{40} \mathrm{Ca}$ & 19.6 & 20.3 & 21.1 \\
\hline
\end{tabular}

We have selected the key nuclei of ${ }^{208} \mathrm{~Pb},{ }^{90} \mathrm{Zr},{ }^{48} \mathrm{Ca}$, and ${ }^{40} \mathrm{Ca}$ spanning a broad range of masses for our analysis. ${ }^{48} \mathrm{Ca}$ and ${ }^{40} \mathrm{Ca}$ are included with a view to augment the range of variation with $A$ and to reinforce the asymmetry component, and thus to facilitate a reasonable dissection of the components in the analysis. As observed in Refs. [12,21,48, Sn isotopes present an anomalous behaviour and are at present problematic theoretically. Sn nuclei being openshell exhibit a significant BCS neutron superfluidity in the ground state thus affecting the Fermi surface significantly. These may require further theoretical considerations than employed so far. With the problem of Sn nuclei not yet understood, we have not included ${ }^{120} \mathrm{Sn}$ isotope in the dissection of finite-size contributions.

The GMR energies for ${ }^{208} \mathrm{~Pb},{ }^{90} \mathrm{Zr},{ }^{48} \mathrm{Ca}$, and ${ }^{40} \mathrm{Ca}$ obtained with the GCM using the parameter sets NL3, SVI-2 and SIGO-c of the three Lagrangian models are given in Table 4. As seen earlier, NL3, SVI-2 and SIGO-c, respectively, produce GMR energies in an increasing order for all the nuclei considered. The energies with NL3 are the lowest, whereas SIGO-c provides the largest values amongst the three Lagrangian models. A preliminary consideration suggests 
that $K_{\infty}$ being the same for these parameter sets, the finite-size contributions to Eq. (2) must be largest with NL3 and smallest with SIGO-c in order to have the noted difference in the GMR energies.

In order to discern which finite-size effect(s) play a crucial role in influencing the GMR energies such as given in Table 4 and portrayed in Figs. 5, we have resorted to the use of Eq. (2). The objective is to see whether a fit to Eq. (2) is possible by relinquishing the aforesaid correlation of $K_{C o u l}$ to $K_{\infty}$. Using the GMR energies given in Table 4 for the parameters sets of the three Lagrangian models, we have performed a $\chi^{2}$-minimization procedure to fit Eq. (2) by treating $K_{\text {surf }}, K_{\text {asym }}, K_{\text {Coul }}$ and $K_{\text {curv }}$ as independent variables. The corresponding $K_{A}$ is evaluated using Eq. (1).

Table 5

The coefficients of expansion (in $\mathrm{MeV}$ ) of $K_{A}$ for the parameter sets of the three Lagrangian models with the same value of $K_{\infty} \sim 272 \mathrm{MeV}$. The last column shows the ratio $K_{\text {surf }} / K_{\infty}$ obtained.

\begin{tabular}{lcccccc}
\hline Model & $K_{\infty}$ & $K_{\text {surf }}$ & $K_{\text {asym }}$ & $K_{\text {Coul }}$ & $K_{\text {curv }}$ & $K_{\text {surf }} / K_{\infty}$ \\
\hline NL3 & 271.8 & $-537 \pm 15$ & $-389 \pm 12$ & $-6.9 \pm 0.2$ & $117 \pm 43$ & -1.98 \\
SVI-2 & 271.4 & $-455 \pm 15$ & $-345 \pm 12$ & $-6.8 \pm 0.2$ & $-52 \pm 45$ & -1.67 \\
SIGO-c & 272.8 & $-273 \pm 17$ & $-395 \pm 13$ & $-7.1 \pm 0.3$ & $-622 \pm 50$ & -1.00 \\
\hline
\end{tabular}

For the purpose of this work, i.e. to investigate the importance of finite-size effects, we fix the value of $K_{\infty}$ to the theoretical one and allow a fit of the GMR energies only to four free parameters, viz., $K_{\text {surf }}, K_{\text {asym }}, K_{\text {Coul }}$ and $K_{\text {curv }}$. It is noted that by doing so it is possible to fit Eq. (2) to the GMR energies well. The results of the fits of the GMR energies for the three Lagrangian models are given in Table 5 . The resulting coefficient $K_{\text {Coul }}$ turns out be similar in value from NL3 to SIGO-c. It is not expected that the Coulomb term would be very different from one Lagrangian model to the other. These values are very close to those which have been obtained using other approaches such as the density-dependent Skyrme forces [20].

It can be seen from Table 5 that by fixing $K_{\infty}$ to the desired value, the surface term can be determined with a considerable precision. The striking feature that emerges from the minimizations is the significant difference in the value of the surface incompressibility. Here $K_{\text {surf }}$ is obtained as -537 $\mathrm{MeV}$ for NL3, $-455 \mathrm{MeV}$ for SVI-2 and $-273 \mathrm{MeV}$ for SIGO-c. The set NL3 provides the largest value of $K_{\text {surf }}$, whereas SIGO-c yields the lowest value. Inevitably, a large difference in the value of $K_{\text {surf }}$ for the three Lagrangian 
models is responsible for the differences in the GMR energies as observed in Figs. 5 and 6. This is also reflected by the significant differences in the ratio $K_{\text {surf }} / K_{\infty}$ shown in the last column. It varies from -1.98 for NL3, -1.67 for SVI-2 to -1.00 for SIGO-c. The ratio with NL3 and SVI-2 is substantially different from the ratio of $\sim-1$ that is obtained from calculations of semiinfinite nuclear matter using the scaling model [42]. On the other hand, a ratio of $\sim-1$ is attained with SIGO-c.

In comparison, the asymmetry coefficient $K_{\text {asym }}$ shows only a limited variation from $\sim-345 \mathrm{MeV}$ to $\sim-395 \mathrm{MeV}$ (Table 5). These values are not far from the value of $-550 \pm 100 \mathrm{MeV}$ obtained in a recent fit of the experimental GMR energies of $\mathrm{Sn}$ isotopes [12]. A value of $-500 \pm 50 \mathrm{MeV}$ has been obtained in a recent analysis of the breathing-mode GMR with the density-dependent Skyrme forces [20].

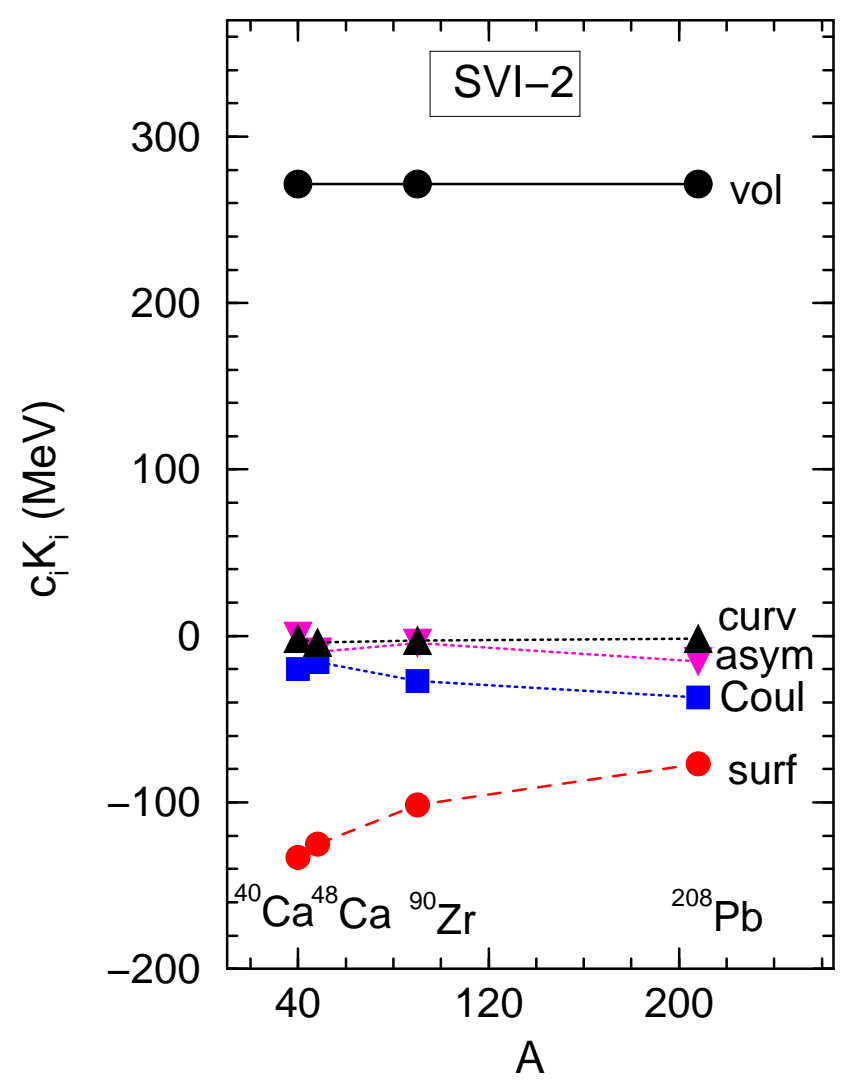

Fig. 6. Contributions of various terms in the expansion $K_{A}=\Sigma c_{i} K_{i}$ using the coefficients $K_{i}$ from Table 5 for the parameter set SVI-2.

For the sake of illustration of the relative magnitudes of various contributions to $K_{A}$ in Eq. (2), we show the size of various terms using the coefficients obtained for the parameter set SVI-2 in Fig. 6. Here we have chosen the set SVI-2 for the sake of illustration. The picture with the other parameters 
sets is similar to it with major differences being in the surface contribution. The curvature term would show differences as maifest in Table 5 especially for SIGO-c. The variation of the Coulomb and the asymmetry terms with the mass number $A$ is relatively small. Additionally, the Coulomb and the asymmetry contributions are an order of magnitude smaller than the surface term.

The surface term provides the largest contribution to $K_{A}$ amongst the finitesize effects. It also shows a significant variation with $A$. Consequently, the surface incompressibility has the ability to modulate the GMR energies of medium mass and lighter nuclei in a significant measure.

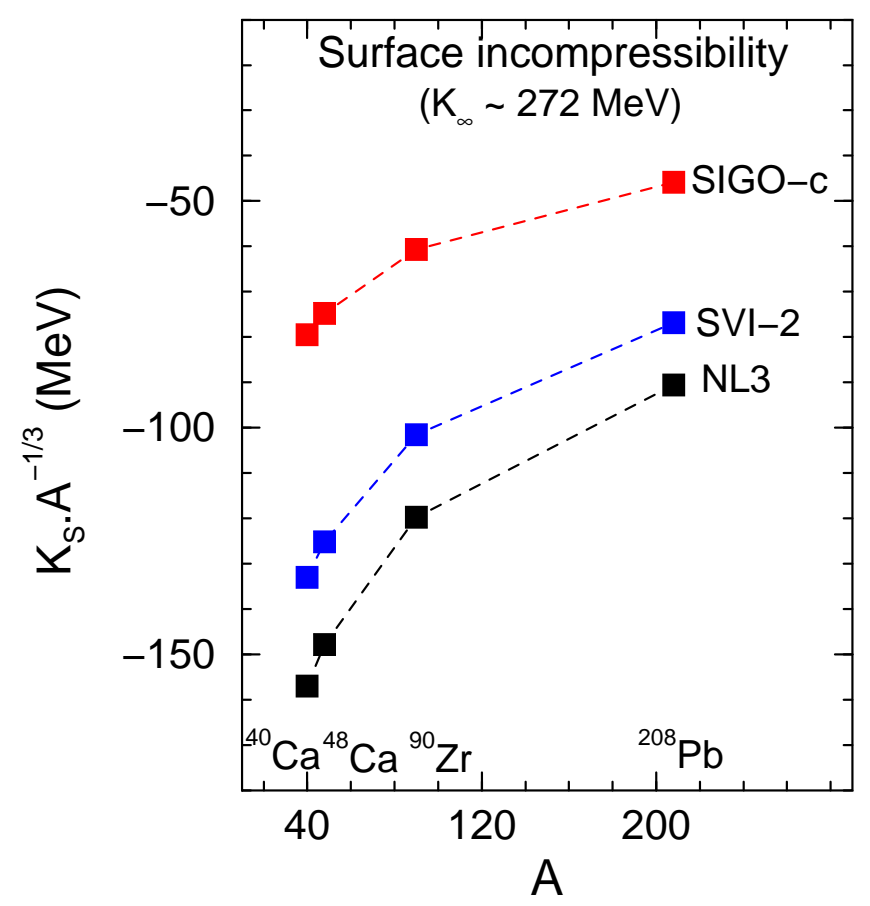

Fig. 7. The surface contribution to $K_{A}$ for the parameter sets NL3, SVI-2 and SIGO-c in the three Lagrangian models having the same value of $K_{\infty} \sim 272 \mathrm{MeV}$.

The relative magnitudes of the surface term for the parameter sets of the three Lagrangian models having the same value of $K_{\infty} \sim 272 \mathrm{MeV}$ are compared in Fig. 7. The surface term exhibits a large variation with mass $A$. Significant differences in the surface term amongst the various Lagrangian models are seen readily in the figure. Inevitably, these differences give rise to the differences in the breathing-mode energies obtained with various Lagrangian models.

Given the relatively smaller magnitude of the Coulomb and the asymmetry terms as seen in Fig. 6, magnitudes of these terms would not show any significant difference from one Lagrangian model to the others. In conclusion, it is the surface term that plays a decisive role in influencing the GMR energies of 
nuclei amongst various Lagrangian models significantly. Whilst the curvature term is not very small especially with SIG-c, it may also be influencing the GMR energies to an extent.

\subsection{Density dependence of the meson masses}

The three Lagrangian models employed in this work are successful in reproducing the ground-state properties of nuclei. Whilst most of the nuclear matter properties e.g., saturation density, saturation binding energy, effective mass and asymmetry energy are not very different from one model to the other, the density dependence of the $\sigma$ and $\omega$ meson masses does show different forms in various models as seen in Section 4 . This difference in the density dependence especially in the sub-saturation densities would influence the surface properties. Consequently, the form of the density dependence of meson masses affects the GMR response implicitly.

The density dependence of meson masses and by implication that of the nuclear interaction in the RMF theory remains an open and challenging problem. The introduction of the nonlinear $\sigma^{3}+\sigma^{4}$ terms in the RMF Lagrangian has led to successful results on ground-state properties of nuclei. It has, however, a major drawback in that the resulting EOS of the NL $\sigma$ model is ubiquitously stiff. The standard NL $\sigma$ sets produce a maximum mass of neutron stars in the vicinity of 2.7-3.0 solar masses. This is well above the spectrum of the observed neutron star masses. Thus, the NL $\sigma$ model is not deemed as suitable for neutron star structure. In view of this, development of other Lagrangian models which could describe finite nuclei as well as nuclear matter at higher densities becomes desirable.

The recent development of the SVI [37] and SIGO [40] models provide a much improved description of nuclear properties vis-a-vis the NL $\sigma$ model. Notably, the SIGO model provides an excellent description of nuclei at the shell closures and also for nuclei in the extreme regions of the periodic table [40]. It also delivers an EOS of nuclear matter which is softer than the NL $\sigma$ counterparts. On the other hand, the Lagrangian model SVI is able to describe the groundstate properties of nuclei without self-interactions of the mesonic fields. It has been shown that introduction of terms of scalar-vector type such as those in SVI would help alleviate the onset of instabilities in nuclear matter [36]. Arguably, the SVI model would also be conducive to a linear realization of chiral symmetry.

Notwithstanding the above, the RMF theory offers alternative possibilities of its density functional to be applicable to nuclei and nuclear matter. A natural question that arises is: what is the density dependence of the meson masses 
that is preferred by nature? At present we do not have an answer to this question. It is difficult to pinpoint as to which Lagrangian model is preferred by experimental data. A comprehensive investigation of the RMF theory with all its attendant problems, virtues and theoretical constraints juxtaposed to a host of experimental data would be necessary in order to be able to unravel the form of the density dependence required by nature.

\subsection{The established parameter sets and confrontation with GMR energies}

In view of the desirability of discerning the density dependence in the RMF theory, it is instructive to examine the results of the GCM calculations on breathing-mode GMR using the standard parameter sets vis-a-vis the available experimental data. The parameter sets which come into such a consideration are the ones which are in the region of physically acceptable values of $K_{\infty}$. This includes the sets NL1 and NL3 of the NL $\sigma$ model, sets SVI-1 and SVI-2 of the SVI model and SIG-OM of the SIGO model. With the exception of NL1, all the other sets have been shown to describe binding energies and charge radii of nuclei along as well as far away from the stability line.

The GMR energies of the key nuclei ${ }^{208} \mathrm{~Pb},{ }^{120} \mathrm{Sn}$ and ${ }^{90} \mathrm{Zr}$ obtained with the GCM approach using the above parameter sets are shown in Table 6. Experimental data on these nuclei are well established and are shown for a comparison. The parameter sets are listed in an ascending order of the GMR energies.

Table 6

The breathing mode GMR energies obtained with constrained GCM calculations using the established parameter sets. The experimental data [10,15] are shown for comparison. The $K_{\infty}$ value (in $\mathrm{MeV}$ ) for the sets is shown in parentheses.

\begin{tabular}{ccccccc}
\hline Sets & NL1 & NL3 & SVI-1 & SVI-2 & SIG-OM & exp. \\
& $(211)$ & $(272)$ & $(264)$ & $(272)$ & $(265)$ & \\
\hline${ }^{208} \mathrm{~Pb}$ & 11.0 & 13.0 & 13.3 & 13.5 & 14.1 & $13.96 \pm 0.28$ \\
${ }^{120} \mathrm{Sn}$ & 12.7 & 15.0 & 15.2 & 15.4 & 16.2 & $15.52 \pm 0.15$ \\
${ }^{90} \mathrm{Zr}$ & 14.1 & 16.9 & 17.2 & 17.5 & 18.2 & $17.81 \pm 0.30$ \\
\hline
\end{tabular}

Without resorting to a disentanglement of $K_{A}$ into its respective components, we compare the GMR energies due to various parameter sets directly with the experimental values. The GMR energies with NL1 are $\sim 3-4 \mathrm{MeV}$ smaller than the experimental values. NL3, on the other hand, provides GMR energies 
which are $\sim 1 \mathrm{MeV}$ smaller than the experimental ones for ${ }^{208} \mathrm{~Pb}$ and ${ }^{90} \mathrm{Zr}$. For ${ }^{120} \mathrm{Sn}$, the difference of the NL3 GMR energy with the experimental value is $\sim 0.5 \mathrm{MeV}$.

In comparison, the SVI-1 GMR energies are slightly bigger than those of NL3 and hence are closer to the experimental values than NL3. However, even with SVI-1, the difference with the experimental data, especially for ${ }^{208} \mathrm{~Pb}$ and ${ }^{90} \mathrm{Zr}$ is not insignificant. On the other hand, the SVI-2 GMR energies for all the three nuclei come much closer to the experimental bounds. As is the case with SVI-1, the GMR energies for ${ }^{208} \mathrm{~Pb}$ and ${ }^{90} \mathrm{Zr}$ with SVI-2 are on the lower side of the experimental values, whereas for ${ }^{120} \mathrm{Sn}$, the SVI-2 value is in good agreement with the experimental datum. Overall, the SVI-2 results show a reasonable good agreement with the experimental data for the three nuclei. The SIG-OM value, in comparison, comes closer to the experimental one for ${ }^{208} \mathrm{~Pb}$. However, SIG-OM overestimates the experimental values for ${ }^{120} \mathrm{Sn}$ by $\sim 0.7 \mathrm{MeV}$ and slightly for ${ }^{90} \mathrm{Zr}$. Thus, the behaviour of $\mathrm{Sn}$ isotope remains anomalous in the GCM approach, as observed in nonrelativistic and relativistic RPA approach [20,21].

From the point of view of the ground-state binding energies and charge radii of nuclei and a good description of data along the stability line and away from it, in conjunction with the breathing-mode GMR energies, the parameter set SVI-2 of the SVI model without self-interactions comes closest to the empirical data. It has to be seen in further exploration of other aspects of nuclear structure and properties as to how close to the data the set SVI-2 will appear. It should be pointed out that this conclusion drawn here is subject to the GCM treatment of the breathing mode GMR. The constrained GCM approach delivers GMR energies slightly smaller than those obtained within the RPA approach. It is worth investigation as to how different the properties of the breathing-mode GMR would appear within the framework of relativistic RPA using various Lagrangian models.

\section{Summary and conclusions}

The breathing-mode GMR is investigated within the framework of the RMF theory using the generator coordinate method. The GMR energy has been calculated for a few key nuclei of ${ }^{208} \mathrm{~Pb},{ }^{120} \mathrm{Sn},{ }^{90} \mathrm{Zr},{ }^{48} \mathrm{Ca}$ and ${ }^{40} \mathrm{Ca}$ covering a broad range of atomic mass. Using the Lagrangian set NL3 of the NL $\sigma$ model, sets SVI-1 and SVI-2 of the SVI model and set SIG-OM of the SIGO model, a paradoxical behaviour of the GMR energies has been found. Contrary to the received wisdom, parameter sets with a higher value of $K_{\infty}$ are observed to yield a lower value of the GMR energy as compared to a higher GMR energy attained with sets having a lower value of $K_{\infty}$. 
In order to resolve the apparent paradox of the GMR energies in the RMF theory, the GMR response has been investigated using the three different Lagrangian models viz., the NL $\sigma$ model, the SVI model and the SIGO model. Employing the constrained GCM approach, GMR energies have been calculated within each Lagrangian model using parameter sets which encompass a broad range of $K_{\infty}$. It is shown that each Lagrangian model exhibits a GMR response that is different from the others. Consequently, each Lagrangian model yields a distinctly different value of the GMR energy for a given value of $K_{\infty}$. The model NL $\sigma$ delivers the lowest GMR energy for a nucleus whereas the model SIGO produces the highest value amongst the three models for any given value of $K_{\infty}$.

The GMR energy is shown to exhibit a sensitivity to the Lagrangian model employed. This behaviour stems from the differences in the implicit density dependence of the meson masses in various models, thus altering the density dependence of the nuclear interaction in the RMF theory from one Lagrangian model to the other. The sensitivity to the density dependence opens up another dimension in the landscape of the GMR energy and incompressibility.

The multiplicity of the GMR energy for a given $K_{\infty}$ in the RMF theory renders the 'microscopic' method of extraction of $K_{\infty}$ using interpolation amongst forces as inapplicable. For this approach to work, however, it is necessary to 'calibrate' the density dependence of the meson masses and correspondingly the nuclear interaction in the RMF theory.

Using the liquid-drop type expansion of the finite nucleus incompressibility $K_{A}$, it is shown that for a given value of $K_{\infty}$, each Lagrangian model yields a markedly different value of the surface incompressibility $K_{\text {surf }}$. The model NL $\sigma$ delivers the largest value of $K_{\text {surf }}$, whereas the model SIGO provides the smallest $K_{\text {surf }}$ amongst the three Lagrangian models considered. Consequently, a different value of the ratio $K_{\text {surf }} / K_{\infty}$ emerges in different models. Different $K_{\text {surf }}$ values arising in various Lagrangian models are shown to be largely responsible for a multitude of GMR energies for a given $K_{\infty}$. Thus, the response of the nuclear surface to compression is found to be dependent on the Lagrangian model employed. Additionally, the ratio $K_{\text {surf }} / K_{\infty}$ with the Lagrangian models NL $\sigma$ and SVI emerges to be significantly larger than the ratio of $\sim-1$ that is usually obtained with the assumption of scaling in the breathing-mode GMR [42].

The GMR response in the non-relativistic Skyrme approach is simple in contrast. The Skyrme approach commonly delivers a GMR energy which depends primarily on the value of $K_{\infty}$ [14]. This difference in the behaviour of the GMR energies between the RMF theory and the Skyrme approach can be attributed to the fact that the form of the density dependence in the Skyrme density functional is well prescribed at the outset in marked contrast to the 
various forms of density dependence which have emerged in the RMF theory. In a forthcoming work, we shall shed light on the differences amongst various Lagrangian models, which produce a varied response to the GMR energy in the RMF theory shown in this work. Further investigations will also be necessary in order to be able to discern and formulate the appropriate density dependence in the RMF theory.

Acknowledgments I thank Lev Savushkin and Roger Hilton for fruitful discussions and for a careful reading of the manuscript. Thanks are due to the University of St. Petersburg Telecommunications, Russia and especially Lev Savushkin for hospitality for my stay in St. Petersburg during my sabbatical leave of absence, where part of this work was carried out. Useful exchanges with S.K. Patra are acknowledged with thanks. 


\section{References}

[1] H.A. Bethe, Rev. Mod. Phys. 62 (1990) 801.

[2] W. Hillebrandt and R.G. Wolff, in Nucleosynthesis Challenges and New Developments, ed. W.D. Arnett and J.M. Truran (Univ. of Chicago, Chicago, $1985)$ p. 131.

[3] E. Baron, H.A. Bethe, G.E. Brown, J. Cooperstein, S. Kahana, Phys. Rev. Lett. 59 (1997) 736.

[4] J.P. Blaizot, Phys. Rep. 64 (1980) 171.

[5] J. Treiner, H. Krivine, O. Bohigas, J. Martorell, Nucl. Phys. A 371 (1981) 253.

[6] M.N. Harakeh and A. van der Woude, Giant Resonances: Fundamental HighFrequency Modes of Nuclear Excitation, Oxford Univ. Press, Oxford (2001).

[7] M. Buenerd, in Proceedings of the International Symposium on Highly Excited States and Nuclear Structure, Orsay, France, 1983, J. Phys. (Paris) Colloq. 45 (1984) C4-115, and references therein.

[8] D.H. Youngblood, P. Bogucki, J.D. Bronson, U. Garg, Y.-W. Lui, C.M. Rosza, Phys. Rev. C 23 (1981) 1997.

[9] H.J. Lu, S. Brandenburg, R. De Leo, M.N. Harakeh, T.D. Poelhekken, A. van der Woude, Phys. Rev. C 33 (1986) 1116.

[10] M.M. Sharma, W.T. A. Borghols, S. Brandenburg, S. Crona, A. van der Woude, M.N. Harakeh, Phys. Rev. C 38 (1988) 2562.

[11] D.H. Youngblood, H.L. Clark, Y.-W. Lui, Phys. Rev. Lett. 82 (1999) 691.

[12] T. Li et al., Phys. Rev. Lett. 99 (2007) 162503.

[13] J.M. Pearson, Phys. Lett. B 271 (1991) 12.

[14] J.P. Blaizot, J.F. Berger, J. DeChargé, M. Girod, Nucl. Phys. A591 (1995) 435.

[15] D.H. Youngblood, Y.-W. Lui, B. John, Y. Tokimoto, H.L. Clark, X. Chen, Phys. Rev. C 69 (2004) 054312.

[16] B.K. Agrawal, S. Shlomo, V. Kim Au, Phys. Rev. C 68 (2003) 031304(R).

[17] J. Piekarewicz, Phys. Rev. C 69 (2004) 041301(R).

[18] P. Gleissl, M. Brack, J. Meyer, P. Quentin, Ann. Phys. (N.Y.) 197 (1990) 205.

[19] M.M. Sharma, W. Stocker, P. Gleissl, M. Brack, Nucl. Phys. A 504 (1989) 337.

[20] H. Sagawa, S. Satoshi, G.-M. Zeng, J.-Z. Gu, X.-Z. Zhang, Phys. Rev. C 76 (2007) 034327. 
[21] J. Piekarewicz, Phys. Rev. C 76 (2007) 031301.

[22] Z.Y. Ma, N. Van Giai, A. Wandelt, D. Vretenar, P. Ring, Nucl. Phys. A 621 (1997) 853.

[23] B.D. Serot, J.D. Walecka, Adv. Nucl. Phys. 16 (1986) 1.

[24] P.G. Reinhard, Rep. Prog. Phys. 52 (1989) 439.

[25] B.D. Serot, Rep. Prog. Phys. 55 (1992) 1855.

[26] P. Ring, Prog. Part. Nucl. Phys. 37 (1996) 193.

[27] Y.K. Gambhir, P. Ring, A. Thimet, Ann. Phys. (N.Y.) 198 (1990) 132.

[28] M.M. Sharma, M.A. Nagarajan, P. Ring, Phys. Lett. B 312 (1993) 377.

[29] G.A. Lalazissis, J. König, P. Ring, Phys. Rev. C 55 (1997) 540.

[30] M.M. Sharma, G.A. Lalazissis, P. Ring, Phys. Lett. B 317 (1993) 9.

[31] M.V. Stoitsov, P. Ring, M.M. Sharma, Phys. Rev. C 50 (1994) 1445.

[32] D. Vretenar, T. Niksic, P. Ring, Phys. Rev. C 68 (2003) 024310.

[33] T. Niksic, D. Vretenar, P. Ring, Phys. Rev. C 66 (2002) 064302.

[34] J. Boguta, A.R. Bodmer, Nucl. Phys. A 292 (1977) 413.

[35] R.J. Furnstahl, C.E. Price, G.E. Walker, Phys. Rev. C 36 (1987) 2590.

[36] A. Sulaksono, T. Mart, T. Bürvenich, J.A. Maruhn, Phys. Rev. C 76 (2007) 041301(R).

[37] M.M. Sharma, Phys. Lett. B 666 (2008) 140.

[38] S.J. Lee, J. Fink, A.B. Balantekin, M.R. Strayer, A.S. Umar, P.G. Reinhard, J.A. Maruhn, W. Greiner, Phys. Rev. Lett. 57 (1986) 2916.

[39] M.M. Sharma, P. Ring, Phys. Rev. C 45 (1992) 2514.

[40] M.M. Haidari, M.M. Sharma, Nucl. Phys. A 803 (2008) 159.

[41] V.N. Fomenko, S. Marcos, L.N. Savushkin, J. Phys. G: Nucl. Part. Phys. 19 (1993) 545.

[42] S.K. Patra, M. Centelles, X. Vinas, M. Del Estal, Phys. Rev. C 65 (2002) 044304.

[43] M.M. Sharma, L.N. Savushkin, (2008, unpublished).

[44] G.E. Brown, M. Rho, Phys. Rev. Lett. 66 (1991) 720.

[45] P. Bonche, J. Dobaczewski, H. Flocard, P.-H. Heenen, Nucl. Phys. A 530 (1991) 149.

[46] Z.Y. Ma, N. Van Giai, H. Toki, Phys. Rev. C 55 (1997) 2385.

[47] Y. Sugahara, H. Toki, Nucl. Phys. A 579 (1994) 557.

[48] U. Garg et al., Nucl. Phys. A 788 (2007) 36c. 